\title{
Awkward trunk postures and their relationship with low back pain in hospital nurses
}

\author{
Maryam Nourollahi ${ }^{\mathrm{a}}$, Davood Afshari ${ }^{\mathrm{b}, *}$ and Iman Dianat ${ }^{\mathrm{c}}$ \\ ${ }^{a}$ Department of Occupational Health Engineering, School of Public Health, Tehran University of Medical \\ Sciences, Tehran, Iran \\ ${ }^{\mathrm{b}}$ Department of Occupational Health Engineering, School of Public Health, Ahvaz Jundishapur University of \\ Medical Sciences, Ahvaz, Iran \\ ${ }^{\mathrm{c} D e p a r t m e n t ~ o f ~ O c c u p a t i o n a l ~ H e a l t h, ~ F a c u l t y ~ o f ~ H e a l t h, ~ T a b r i z ~ U n i v e r s i t y ~ o f ~ M e d i c a l ~ S c i e n c e s, ~ T a b r i z, ~ I r a n ~}$
}

Received 27 September 2016

Accepted 4 August 2017

\begin{abstract}
.
BACKGROUND: Low back pain (LBP) is the most common work-related musculoskeletal problem among healthcare workers including nurses. Awkward trunk postures have been recognized as one common problem in this group.

OBJECTIVE: The aims of this study were to: a) continuously assess trunk postures for an entire shift work in various hospital wards, and b) examine the relationship between the duration of exposure to awkward trunk postures and the occurrence of low back pain (LBP) among nurses.

METHODS: Eighty nurses from eight wards in a hospital participated in this cross-sectional study. The prevalence of LBP was determined using the revised Nordic Musculoskeletal Questionnaire. Full-shift work trunk posture exposure was measured using an inclinometer.

RESULTS: The prevalence of LBP among hospital nurses was $72 \%$. The highest percentage of time spent in awkward postures in the sagittal plane (trunk flexion $\geq 45^{\circ}$ ) and in the frontal plane (lateral bend $\geq 20^{\circ}$ ) was recorded in the general $(65.6 \% \pm 12.2)$ and orthopedic $(48.4 \% \pm 7.4)$ wards, respectively. Logistic regression analyses showed that the duration of exposure to awkward trunk postures had a significant relationship with LBP $(P<0.05)$.

CONCLUSIONS: The results highlight the potential risks of nursing job in terms of frequent and extreme trunk awkward postures, which may lead to the development of LBP. The findings can help to develop guidelines regarding prioritizing ergonomic interventions to reduce the prevalence of LBP among hospital nurses.
\end{abstract}

Keywords: Inclinometer, spinal, physical workload, musculoskeletal disorders

\section{Introduction}

The most common work-related musculoskeletal disorder (WRMSD) among healthcare workers

*Address for correspondence: Davood Afshari, Department of Occupational Health, School of Public Health, Ahvaz Jundishapur University of Medical Sciences, P. O. Box: 61355-131, Ahvaz, Iran. Tel.: +98 613373 8269; Fax: +98 613373 8282; E-mails: Afshari@ajums.ac.ir and davodafi@yahoo.com. is low back pain (LBP) [1-4]. Patient care tasks have been identified as the most frequent cause of WRMSDs, especially in the back and the lumbar region. Nursing staff with patient-handling tasks have a high prevalence rates of lumbar region injuries [5]. The prevalence of LBP among hospital nurses in Iran has been reported to be between $30 \%-60 \%$ [6-8]. Activities of nurses in patient-handling tasks require a considerable physical capacity. As far as the 
prevalence of WRMSDS is concerned, healthcare workers rank second only after industrial jobs in terms of their physical work load [9-11].

Despite the existence of advanced equipment and facilities for patient treatment in Iranian hospitals, the ergonomic and health-related issues of healthcare workers are commonly not given sufficient consideration. A number of factors including ergonomically untrained personnel, lack of appropriate methods of manual handling and transferring patients, inappropriate layout of workstations and improper devices for transferring patients impose heavy physical workloads on these personnel.

Epidemiological studies have shown that awkward postures, heavy manual handling and transferring of patients, repetitive movements, monotonous tasks, and standing positions for prolonged hours are ergonomic risk factors associated with nursing jobs $[10,12,13]$. A review of high-risk tasks in this occupation specifies that most tasks require awkward postures during various activities, and nurses sometimes need to perform tasks for a prolonged period with a flexed trunk. Awkward postures are known to be the most common and most significant factors associated with the occurrence of musculoskeletal disorders among nurses [6, 12, 14]. It has been reported that awkward postures and frequent heavy lifting have a particularly significant effect on workers' low back pain [14-16].

One of the important factors that can have an important contribution to risk management of low back injuries is to determine frequency and duration of exposure to stressful postures. Studies have shown that a combination of awkward postures, external loads, and the frequency and duration of high-risk tasks such as transferring or lifting a patient may well represent the most significant mechanical loads on the spine [17].

These risk factors must be assessed in order to manage the risk of back injuries. This can be determined by using various tools and methods such as questionnaires, videotaping techniques, and/or direct measurement techniques. Each of these tools has its own advantages and disadvantageous. The use of videotaping techniques to determine risk factors can be extremely difficult, mainly because the tasks of nursing staff are not cyclical and are not limited to a specific workstation. Meanwhile, privacy issues in relation to personnel and patients make the observational method much more difficult. Therefore, evaluations should be performed through questionnaires or by means of direct measurements.
Generally, it has been proven that the use of selfreporting procedures for the determination of risk factors is not accurate in the evaluation of musculoskeletal disorders [18].

Epidemiological studies have recommended the use of direct measurement as a suitable device for accurate assessment of postures. The primary advantages of direct measurement techniques over observational methods are their higher rates of accuracy, lower costs, and higher levels of reliability [18, 19]. Recent studies have proven that some direct measurement techniques are more affordable than observational methods [18]. Studies have been conducted to date about musculoskeletal problems of Iranian nurses have been qualitative studies based on self-reports and observational techniques, where risk factors have been identified based entirely on qualitative methods [20-22]. Thus, the present study was conducted to: a) continuously assess trunk postures for an entire shift work in various hospital wards based on direct measurement; and b) examine the relationship between the duration of exposure to awkward trunk postures and the occurrence of LBP in hospital nurses. The results of this study are expected to provide a comprehensive and detailed basis for ergonomic interventions required in various hospital wards.

\section{Methods}

\subsection{Participants}

Eighty hospital nurses from eight hospital wards (ICU, CCU, orthopedic, internal, emergency, neurology, surgical, maternity and gynecology wards) in the city of Tehran-Iran participated in this crosssectional study. The inclusion criteria for the study were as follows: ages 30 to 42 years and work experience $>3$ years. The exclusion criteria were having previous spinal surgery and a significant vertebral deformity resulting from any etiology.

\subsection{Data collection}

The prevalence of musculoskeletal disorders experienced by the participants during the past year was evaluated using the validated Farsi version of Nordic questionnaire. Demographic details of the study participants were also recorded. These data were collected during an interview process. 
Full-shift work trunk posture (flexion-extension and left-right lateral bend) exposure was measured using a tri-axial accelerometer with a built-in data logger called the Virtual Corset (VC) (Microstrain, Inc., VT, USA). The VC is a wireless, batterypowered, pager-sized portable logger with a $2 \mathrm{MB}$ of onboard memory and a total weight of $72 \mathrm{~g}$.

Upon completion of sample duration, postural data were downloaded to a PC for analysis. Prior to data analysis, VC signals were filtered with a low pass of $3 \mathrm{~Hz}$ and 4 th order Butterworth filter. The trunk angles were calculated using amplitude probability distribution functions (APDF) in custom Matlab (R2013a, Mathworks, Natick, MA) software. The 10th, 50th, and 90th percentiles of the APDF are referred to as "low," "median" and "peak" angles. Limits for "neutral" and "extreme" postures were defined according to the draft standard on working postures [23]. All intended parameters were calculated using custom programs developed in Matlab R2007B (The Math Works Inc., Natick, MA, USA).

\subsection{Statistical analyses}

All statistical analyses were performed using SPSS 16.0 (SPSS Inc.). Logistic regression analyses with their corresponding 95\% Confidence Intervals (CIs) were used to evaluate the relationship between the awkward trunk postures and the occurrence of LBP. $P$ values $<0.05$ was considered as statistically significant.

\section{Results}

Study population consisted of 80 females aged 30-42 years. Table 1 shows the characteristics of study participants. The highest prevalence of musculoskeletal symptoms was related to LBP, so that $72 \%$ of the sample reported this (as shown in Table 2).

Table 3 shows the trunk inclination angles in the sagittal and frontal planes for participants working in different wards. Overall, the highest 90th percentile trunk flexion angle was measured in the general and

Table 1

Demographic characteristics of the study population $(n=80)$

\begin{tabular}{lcc}
\hline Variables & Mean & $\mathrm{SD}$ \\
\hline Age (years) & 38.2 & 8.1 \\
Job tenure (years) & 7.1 & 4.9 \\
BMI $\left(\mathrm{kg} / \mathrm{m}^{2}\right)$ & 21.8 & 2.65 \\
Daily working hours & 9.4 & 1.2 \\
\hline
\end{tabular}

Table 2

Prevalence of musculoskeletal disorders in different body regions of hospital nurses $(n=80)$

\begin{tabular}{lc}
\hline Body region & N (percent) \\
\hline Lower back & $58(72)$ \\
Upper back & $46(57)$ \\
legs & $49(61)$ \\
Hands/wrists & $44(55)$ \\
Neck & $37(46)$ \\
Shoulders & $55(42)$ \\
Knees & $50(62)$ \\
Elbows & $24(30)$ \\
Hip & $17(21)$ \\
\hline
\end{tabular}

the ICU wards, with the mean 90th percentile flexion angles of $89^{\circ}$ and $88^{\circ}$, respectively. Meanwhile, the highest 90th percentile of trunk angle in the frontal plane was measured in the general and orthopedic wards, with the mean 90th percentile lateral angles of $33^{\circ}$ and $29.6^{\circ}$, respectively. The highest and lowest percentages of time spent in awkward postures in the sagittal plane $\left(\geq 45^{\circ}\right)$ were measured in participants working in the general $(65.6 \% \pm 12.2)$ and maternity $(13.1 \% \pm 2.4)$ wards, respectively; while these values for the frontal plane $\left(\geq 20^{\circ}\right)$ were recorded among participants working in the orthopedic $(48.4 \% \pm 7.4)$ and surgical (19.1\% \pm 2.7 ) wards (Fig. 1). The results of logistic regression showed that there is a significant relationship between the prevalence of LBP and duration of exposure to awkward postures in the sagittal and frontal planes $(P<0.05)$ (Table 4$)$.

\section{Discussion}

The prevalence of MSDs was investigated among nurses with the results showing a high 12-month prevalence of MSD symptoms affecting the upper body; the highest rates were recorded for the lower back. According to Survey of Iran National Health (2012), low back pain was a prevalent disorder by $\% 29$ in the general population of Iran while it was prevalent by $\% 72$ in nurses in the present study [24]. This indicates that nursing can be considered as a high risk occupation for developing low back disorders.

A number of qualitative epidemiological studies have demonstrated that in most private and public hospitals in Iran, basic ergonomic principles are not applied in the design of workstations and in the transportation of patients $[6-8,22]$. A number of previous studies have examined the relationships of demographic data and ergonomic risk factors with the prevalence of WRMSDs through self-reporting 
Table 3

Mean (SD) of trunk inclination and lateral inclinations summary measures among hospital nurses

\begin{tabular}{|c|c|c|c|c|c|c|c|c|c|}
\hline Exposure variables & ICU & Orthopedic & General & $\mathrm{CCU}$ & Emergency & Neurology & Surgery & Maternity & Gynecology \\
\hline \multicolumn{10}{|l|}{ Lateral bend angle } \\
\hline $10\left(^{\circ}\right)$ & $8.6 \pm 1.6$ & $11.8 \pm 1.3$ & $11 \pm 0.89$ & $5.4 \pm 1.6$ & $2.3 \pm 1.6$ & $4.5 \pm 1.3$ & $1.6 \pm 0.84$ & $2.5 \pm 1.3$ & $1.7 \pm 1.2$ \\
\hline $50\left({ }^{\circ}\right)$ & $19.9 \pm 3.2$ & $21.8 \pm 2.8$ & $22.3 \pm 3.5$ & $10.7 \pm 2.9$ & $10.4 \pm 1.5$ & $13.5 \pm 2$ & $2 \pm 1.1$ & $5.9 \pm 1.5$ & $7.2 \pm 1.1$ \\
\hline $90\left(^{\circ}\right)$ & $29 \pm 5.2$ & $29.9 \pm 7.4$ & $33.6 \pm 3.1$ & $21.5 \pm 3.2$ & $16.7 \pm 2.8$ & $23.5 \pm 1.2$ & $19.1 \pm 2.7$ & $15.3 \pm 3.5$ & $17.6 \pm 2.5$ \\
\hline \multicolumn{10}{|l|}{ Flexion angle } \\
\hline $10\left(^{\circ}\right)$ & $18.7 \pm 7.3$ & $18.9 \pm 4.4$ & $13.8 \pm 3.3$ & $5.4 \pm 5.6$ & $16 \pm 3.4$ & $15 \pm 4.2$ & $14.8 \pm 5.8$ & $10.2 \pm 5.3$ & $2.0 \pm 3.8$ \\
\hline $50\left({ }^{\circ}\right)$ & s $53 \pm 9.5$ & $43.4 \pm 5.1$ & $48.6 \pm 4.1$ & $30.9 \pm 4.2$ & $30.8 \pm 2.8$ & $26.3 \pm 4.4$ & $16.3 \pm 9$ & $18.7 \pm 4.9$ & $14.8 \pm 7.2$ \\
\hline $90\left({ }^{\circ}\right)$ & $87.9 \pm 14.7$ & $80.2 \pm 14.3$ & $89 \pm 15.8$ & $63.1 \pm 8.9$ & $63.2 \pm 4.6$ & $58.7 \pm 6.9$ & $43.4 \pm 7.1$ & $38.1 \pm 4.6$ & $32.9 \pm 8.3$ \\
\hline
\end{tabular}

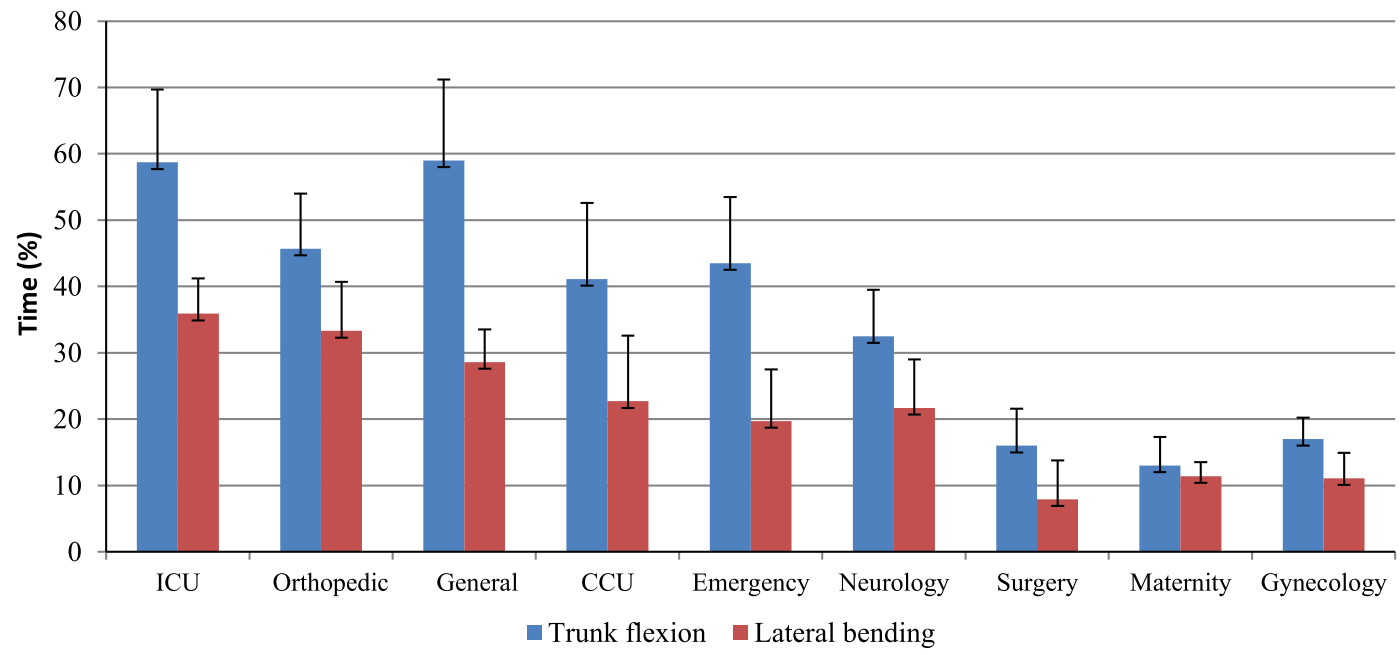

Fig. 1. The mean (SD) percentage of work time with the trunk flexed $\geq 45^{\circ}$ and lateral bending $\geq 20^{\circ}$ among hospital nurses.

techniques. However, since these techniques are incapable of accurately predicting the risk factors, their outcomes may not be accurate and the resultant guidelines for ergonomic interventions may be questionable. The present study is one of the first attempts to evaluate back postures, as one of the major risk factors contributing to LBP, through continuous monitoring of trunk postures during an entire shift in hospital nurses.

As shown in this research, most of the study participants exposed to frequent high-risk and complex tasks, although the percentage of time spent in extreme postures varied according to their wards. This may not be surprising given the variety of physically demanding tasks that hospital nurses have to deal with during their routine activities. The results indicated that the median and peak trunk flexion angles of nurses working in the general, orthopedic and the CCU wards was generally higher than those of other participants. The peak flexion angles in the general, orthopedic and an ICU ward ranged between $80^{\circ}$ to $88^{\circ}$, which is considerable. Laboratory studies have also highlighted the significance of forward flexion angles on compressive spinal loads as well as the importance of flexion angles on spinal loading. For example, an increased trunk flexion angle from the upright position to approximately $70^{\circ}$ with a $20 \mathrm{~kg}$ load can increase intradiscal pressure at L4-L5 by up to $230 \%$ [17]. It is therefore necessary to adopt appropriate ergonomic interventions in the workplace

Table 4

Relationship exposure time to awkward postures and low back pain among hospital nurses

\begin{tabular}{|c|c|c|c|c|}
\hline Trunk/Lateral inclination angle & \multicolumn{2}{|c|}{ LBP } & \multirow[t]{2}{*}{$P^{*}$} & \multirow[t]{2}{*}{ OR $(95 \% \mathrm{CI})$} \\
\hline Trunk inclination angle & Yes & No & & \\
\hline $\begin{array}{l}\text { Time in extreme posture }(\geq 45)(\%) \\
\text { Lateral inclination angle }\end{array}$ & $54.6 \pm 22.3$ & $27.1 \pm 19$ & 0.018 & $1.34(1.22-2.14)$ \\
\hline Time in extreme posture $(\geq 20)(\%)$ & $39.5 \pm 21.7$ & $19.8 \pm 25$ & 0.015 & $1.45(1.12-2.05)$ \\
\hline
\end{tabular}


to reduce the frequency and duration of exposure to awkward postures in this occupational group.

The frequency of patient-transfer tasks was also varied between the wards. Wards with a high proportion of mobile patients such as maternity or/and gynecology had only a few transfers, probably because the patients could move independently in bed and leave the bed without help. On the other hand, employees working in the orthopedic ward were more frequently exposed to high physical pressure while handling and transferring patients with severe fractures. In the orthopedic ward, patient-handling tasks including transferring a patient between a bed and a stretcher, transferring a patient from a chair to a bed, holding a patient's extremities, and preparing a limb could lead to awkward postures and increased physical workload. Past research has also confirmed that patient-handling tasks can lead to higher proportion of awkward postures than non-patient-handling tasks [25].

The results of this study showed a significant relationship between the percentage of work time spent in trunk flexion angles exceeding $45^{\circ}$ and the occurrence of LBP among nurses. The participants working in the ICU, general, and orthopedic wards had the longest duration of exposure to awkward postures. For example, nurses working in the ICU ward spent more than half of their work time $(58.7 \%)$ with trunks flexed at greater than $45^{\circ}$. Neumann et al. [26] reported that nurses working in an extreme trunk flexion position (e.g. performing manual lifting tasks while assisting patients with mobility needs) were at high risk of low back disorder. In the above mentioned wards, the frequency of high-risk tasks (such as manual handling and transferring of patients from stretchers to beds) was higher than other wards, and therefore it is expected that the duration of exposure to awkward postures would be correspondingly increased.

When it comes to awkward postures, nonadjustability of hospital beds and the lack of match between bed heights and anthropometric dimensions (height and reach limits) of employees should also be taken into account. In fact, "vertical height a patient is lifted" and "distance a patient is moved" are important factors in terms of biomechanical principles. This is important because mismatch between anthropometric characteristics and the dimensions of a bed may lead to awkward postures, and consequently to increased spinal load [27]. It has been suggested that tasks with low vertical height and long horizontal distances of hands from body can lead to awkward postures (trunk flexion angle $\geq 45^{\circ}$ ), and consequently generate L4-L5 compression loads that approach or exceed the limit of $3400 \mathrm{~N}$ [28].

The height of the majority of beds in the studied hospital was adjusted manually and/or was nonadjustable. Thus, this could result in unnecessary awkward (flexed) trunk postures even for performing simple tasks such as measuring blood pressure, injecting medicine, replacing pads, etc. Such conditions can increase the sustained trunk flexion and workload, particularly in some wards such as the ICU and internal wards, which need further services. The results of a study by Freitag et al. (2007) among nurses indicated that a total of $38 \%$ of awkward postures were related to bed tasks such as bed making and basic care, and therefore correct adjustment of bed height might have a major effect on the frequency of awkward postures [16]. Caboor et al. [29] shown in laboratory experiments that adjustment of the bed height can significantly reduce the awkward postures among nurses [29].

The median (50th percentile) trunk flexion angle among nurses in the $\mathrm{CCU}$, maternity and neurology wards was between $26^{\circ}$ to $30^{\circ}$, which indicates that the workload is low. On the other hand, the studied nurses in these wards spent $30 \%$ to $40 \%$ of their work time with extreme trunk flexion $\left(>45^{\circ}\right)$. One important factor that has a major impact on LBP risk management is to determine the duration of exposure to stressful postures. Based on the literature, longterm activity with awkward postures for 20 minutes or more can lead to the development of the mental and physical fatigue. Furthermore, the changes in the soft tissues of the trunk has been reported after spending 5-20 minutes of constant trunk flexion [30].

This study indicated that physical workload varies among nurses depending on the nature and variety of their task demands, which can consequently be specified as frequency and duration of exposure to extreme postures. Appropriate and comprehensive planning is therefore needed to control and prevent back injuries resulting from stressful postures in all wards, especially in high-risk ones. Therefore, primary control and intervention plans aimed at reducing stressful postures should focus on the following areas:

- Equipping wards with mechanical and/or electromechanical devices for manual handling and transferring of patients.

- Providing guidelines regarding manual handling and transferring of patients for those employees involved in such activities. 
- Providing appropriate planning and scheduling for the maintenance of patient transfer equipment.

- Providing appropriate planning for the reduction of exposure to stressful postures, especially in high-risk wards, through rotating shift work in wards with lower high-risk tasks.

\subsection{Limitations}

In this research was only to explore any relationship between back postures and the prevalence of low back pain in nurses, in order to determine more precisely the risk factors in each ward of the hospital, further study is recommended to assess the mechanical loads on the back and physiological demands associated with each task in nurses.

Axial rotation of the trunk is associated with pain disorders affecting the back, this is an important consideration. A second limitation was the exclusion of rotation as a movement variable; this plane of movement was excluded because of limitations inherent to the inclinometer. Since little lateral bending occurred, it may be that nurses relay on rotational movements to shift their visual field.

\section{Conclusion}

This is one of the first attempts that quantitatively evaluated the work postures of nurses performing tasks throughout the duration of shift work in various wards of a hospital in Iran. The results indicated a high prevalence of LBP among nurses, which was associated with the duration of exposure to awkward trunk postures. Although the majority of tasks performed by the hospital nurses were relatively similar, their physical workload was different which may be attributed to the frequency and duration of exposure to extreme postures. The findings have important implications for prioritizing ergonomic interventions for reducing the prevalence of LBP among hospital nurses.

\section{References}

[1] Trinkoff AM, Lipscomb JA, Geiger-Brown J, Storr CL, Brady BA. Perceived physical demands and reported musculoskeletal problems in registered nurses. American Journal of Preventive Medicine. 2003;24(3):270-5.

[2] Menzel NN, Brooks SM, Bernard TE, Nelson A. The physical workload of nursing personnel: Association with musculoskeletal discomfort. International Journal of Nursing Studies. 2004;41(8):859-67.

[3] Smith DR, Sato M, Miyajima T, Mizutani T, Yamagata Z. Musculoskeletal disorders self-reported by female nursing students in central Japan: A complete cross-sectional survey. International Journal of Nursing Studies. 2003;40(7): 725-9.

[4] Serranheira F, Sousa-Uva M, Sousa-Uva A. Hospital nurses tasks and work-related musculoskeletal disorders symptoms: A detailed analysis. Work. 2015;51(3):401-9.

[5] Tamminen-Peter L. The physical load of the movement assistance of the elderly on nurses: A comparison of three transfer methods. Turku Regional Institute for Occupational Health Final report of the Finnish Work Environment Fund. 2002.

[6] Afshari D, Mohammadi A, Saki A, Movafaghpour M. Continuous monitoring of back postures using portable inclinometer among nursing assistants. Iran Occupational Health. 2014;11(3):30-9.

[7] Abdollahzade F, Mohammadi F, Dianat I, Asghari E, Asghari-Jafarabadi M, Sokhanvar Z. Working posture and its predictors in hospital operating room nurses. Health Promot Perspect. 2016;(6)17-22.

[8] Arsalani N, Fallahi-Khoshknab M, Josephson M, Lagerström M. Musculoskeletal disorders and working conditions among Iranian nursing personnel. International Journal of Occupational Safety and Ergonomics. 2014;20(4):671-80.

[9] Engels JA, Van Der Gulden J, Senden TF, van't Hof B. Work related risk factors for musculoskeletal complaints in the nursing profession: Results of a questionnaire survey. Occupational and Environmental Medicine. 1996;53(9):636-41.

[10] Smedley J, Egger P, Cooper C, Coggon D. Manual handling activities and risk of low back pain in nurses. Occupational and Environmental Medicine. 1995;52(3):160-3.

[11] Robstad Andersen G, Westgaard RH. Perceived occupational exposures of home care workers and the association to general tension, shoulder-neck and low back pain. Work. 2014;49(4):723-33.

[12] Hodder JN, Holmes MW, Keir PJ. Continuous assessment of work activities and posture in long-term care nurses. Ergonomics. 2010;53(9):1097-107.

[13] Village J, Frazer M, Cohen M, Leyland A, Park I, Yassi A. Electromyography as a measure of peak and cumulative workload in intermediate care and its relationship to musculoskeletal injury: An exploratory ergonomic study. Applied Ergonomics. 2005;36(5):609-18.

[14] Hodder JN, Holmes MWR, Keir PJ. Continuous assessment of low back loads in long-term care nurses. Ergonomics. 2010;53:1097-107.

[15] Daynard D, Yassi A, Cooper J, Tate R, Norman R, Wells R. Biomechanical analysis of peak and cumulative spinal loads during simulated patient-handling activities: A substudy of a randomized controlled trial to prevent lift and transfer injury of health care workers. Applied Ergonomics. 2001;32(3):199-214.

[16] Freitag S, Ellegast R, Dulon M, Nienhaus A. Quantitative measurement of stressful trunk postures in nursing professions. Annals of Occupational Hygiene. 2007;51(4):385-95.

[17] Arjmand N, Gagnon D, Plamondon A, Shirazi-Adl A, Larivi ere C. A comparative study of two trunk biomechanical models under symmetric and asymmetric loadings. J Biomech. 2010;43:485-91.

[18] Trask C, Teschke K, Village J, Chow Y, Johnson P, Luong N, et al. Measuring low back injury risk factors in challenging work environments: An evaluation of cost and feasibility. 
American Journal of Industrial Medicine. 2007;50(9): 687-96.

[19] Hansson G, Asterland P, Holmer N-G, Skerfving S. Validity and reliability of triaxial accelerometers for inclinometry in posture analysis. Medical and Biological Engineering and Computing. 2001;39(4):405-13.

[20] Abedini R, Choobineh A, Hasanzadeh J. Patient manual handling risk assessment among hospital nurses. Work. 2015;50(4):669-75.

[21] Sadeghian F, Coggon D, Ntani G, Hosseinzadeh S. Predictors of low back pain in a longitudinal study of Iranian nurses and office workers. Work. 2015;51(2):239-44.

[22] Morshedi R, Boazar M, Afshari D. Biomechanical analysis of manual lifting of loads and ergonomics solutions for nursing assistants. Journal of Ergonomics. 2015;3(1):17-24.

[23] Standard B. Safety of machinery: Human physical performance. BS EN. 2005:1005-4.

[24] Biglarian A, Seifi B, Bakhshi E, et al. Low back pain prevalence and associated factors in Iranian population: Findings from the national health survey. Pain Res Treat. 2012;2012: 5-15.

[25] Hignett S. Postural analysis of nursing work. Applied Ergonomics. 1996;27(3):171-6.
[26] Neumann WP, Wells RP, Norman RW, Andrews DM, Frank J, Shannon HS, et al. Comparison of four peak spinal loading exposure measurement methods and their association with low-back pain. Scandinavian Journal of Work, Environment \& Health. 1999;404-9.

[27] Merryweather AS, Morse JM, Doig AK, Godfrey NW, Gervais P, Bloswick DS. Effects of bed height on the biomechanics of hospital bed entry and egress. Work. 2015;52(3):707-13.

[28] Arjmand N, Amini M, Shirazi-Adl A, Plamondon A, Parnianpour M. Revised NIOSH Lifting Equation May generate spine loads exceeding recommended limits. International Journal of Industrial Ergonomics. 2015;47:1-8.

[29] Caboor D, Verlinden M, Zinzen E, Van Roy P, Van Riel M, Clarys JP. Implications of an adjustable bed height during standard nursing tasks on spinal motion, perceived exertion and muscular activity. Ergonomics. 2000;43(10):1771-80.

[30] Beach TA, Parkinson RJ, Stothart JP, Callaghan JP. Effects of prolonged sitting on the passive flexion stiffness of the in vivo lumbar spine. The Spine Journal. 2005;5(2):145-54. 